\title{
DEVELOPMENT OF A FIRE DAMAGE INDEX FOR IMMOVABLE CULTURAL HERITAGE
}

\author{
L. GERARDO F. SALAZAR ${ }^{1 *}$, ESMERALDA PAUPÉRIO ${ }^{2}$ AND XAVIER ROMÃO ${ }^{3}$ \\ ${ }^{1}$ CONSTRUCT-LESE, Faculty of Engineering - University of Porto (FEUP) \\ Rua Dr. Roberto Frias, 4200-465Porto, Portugal \\ Email: gsalazar@fe.up.pt \\ ${ }^{2}$ Construction Institute, CONSTRUCT-LESE, Faculty of Engineering - University of Porto (FEUP) \\ Rua Dr. Roberto Frias, 4200-465 Porto, Portugal \\ Email: pauperio@fe.up.pt \\ ${ }^{3}$ CONSTRUCT-LESE, Faculty of Engineering - University of Porto (FEUP) \\ Rua Dr. Roberto Frias, 4200-465 Porto, Portugal \\ Email: xnr@fe.up.pt
}

Keywords: Fire Damage Impact Index, Immovable Cultural Heritage, Vulnerability Assessment

\begin{abstract}
Available fire risk assessment approaches are mostly developed for new buildings. These approaches encompass minimum provisions defined by standards that are valid in certain countries, and few of these methods were thought exclusively for assessing the fire safety of cultural heritage. Given the diversity and specificity of cultural heritage assets, simple approaches are thus required to analyse their fire safety and establish their risk mitigation needs. In this context, a simplified fire damage index dedicated to assessing the fire vulnerability of immovable cultural heritage assets is proposed. The proposed index is the result of a weighted multi-parameter evaluation that can be correlated with the level of damage that the cultural heritage asset (including its contents) is expected to suffer under a fire. The proposed index involves twenty-one indicators divided into four categories and offers a flexible approach for universal applicability. The performance of the proposed approach is illustrated for two case studies in Portugal. The results highlight the advantages of having a simple methodology that can be used for the preliminary risk analysis of a large number of assets to identify those requiring risk mitigation measures or detailed and resource-demanding analyses.
\end{abstract}

\section{INTRODUCTION}

Fires that affected cultural heritage assets in recent years (e.g. the National Museum of Rio de Janeiro (Brazil) in 2018, and of the Cathedral of Notre Dame (France) in 2019) emphasise the need for more studies related to their fire safety. The occurrence of these fires can be connected to various factors ranging from a general lack of maintenance, to a lack of adequate safety measures during restoration works. Moreover, historic constructions exhibit a higher fire vulnerability since they were constructed without considering fire preparedness measures, namely aspects commonly considered in current construction standards such as adequate evacuation routes or emergency exits. The combustible nature of many cultural heritage elements often forces stakeholders to address these issues by integrating modern fire protection 
systems in historic constructions. However, the integration of such systems needs careful consideration to avoid the loss of cultural heritage values due to their installation, as well as the occurrence of further damage during their use (e.g. water sprinkler systems in scenarios involving cultural heritage assets sensitive to water damage).

Several methods have been developed to assess fire risk in buildings, but most of the robust approaches were developed for new buildings and involve formulations that are not compatible with cultural heritage values or are exclusively for one type of heritage. The development of a robust fire safety assessment method for multiple cultural heritage assets is thus needed, namely one that accounts for both the building and its cultural contents, and that can be implemented in any country. To address this need, a fire damage impact index of vulnerability is proposed herein. The index is presented in the following and some of its components are detailed for the case of single cultural heritage assets (i.e. assets physically separated from other assets or constructions) that can host movable heritage or have heritage elements attached to the main asset. Typical examples of such heritage assets are historic houses, religious buildings or museums. Following the description of the proposed index, an application to two real cases with different expected damage impacts if case of fire is also presented.

\section{FIRE DAMAGE INDEX METHODOLOGY}

The proposed index was developed following a thorough literature review focusing, in particular, methods that gave more emphasis to historical constructions. The indicators selected for the fire index were determined by examining international statistics concerning the causes of multiple fires, existing fire risk assessment methods, as wel providing guidance on seen to generally be in for historical constructions in case of fire, ranked in three tevels (i.e. ligh heavy $\left.\left(70 \leq D_{Y} \leq 100\right)\right)$. Light damage refers to cases where the cultural asset is expected to

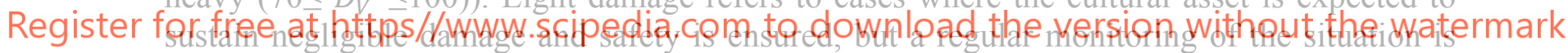

recommended. Medium damage refers to situations where the cultural asset can suffer damage that may be partially recovered in case of fire. Heavy damage refers to cases that may involve irreparable damage or the total loss of the cultural asset. The proposed index is compatible with the Level of Vulnerability $\left(V_{L}\right)$ assessment proposed by [8] that integrates a factor of easiness of reparation and restoration $\left(R_{f}\right)$ and a heritage value factor $\left(B V_{f}\right)$ and can be defined as:

$$
V_{L}=D_{V} \times R_{f} \times B V_{f}
$$

The fire index establishes the relevance of all the twenty-one indicators divided into four groups $\left(G_{i}\right)$ (building properties (BP); utilities: electric power, gas and telecommunications (UEPGT); firefighting measures (FM); emergency preparedness planning (EPP)). Each indicator is assigned to one of five damage impact classes (very low (A), low (B), moderate (C), high (D), and very high (E)). The classification of a group corresponds to that of the indicator that exhibits the worst performance. This assumes that the bad performance of one indicator within a group is enough to intensify the susceptibility of having an ignition, the fire propagation or the difficulty to fight fire. The classes $\left(C_{i}\right)$ are then scored with values of $0(\mathrm{~A})$, 25 (B), 50 (C), 75 (D), 100 (E). The final index is then obtained by a weighted combination of 
the classification of each group. Based on the literature review, weights of $35 \%, 20 \%, 30 \%$ and $15 \%$ were defined for BP, UEPGT, FM and EEP, respectively.

\subsection{Building Properties (BP)}

Indicators of this group refer to different aspects related to the physical properties of the building (material properties, geometrical properties, spaces and their separation, the existence of combustible materials), except installations considered in the UEPGT, and to its immediate surrounding area. Physical properties are relevant for fire risk assessment in terms of their fire resistance, combustibility and the amount of fire load that they represent (considering movable contents and building materials). Moreover, spaces (corridors, halls, rooms, etc.) and their separation provide information related to fire propagation. Indicators in this group are the fire load density $\left(P_{1}\right)$, the fire resistance $\left(P_{2}\right)$, the compartmentalisation $\left(P_{3}\right)$, the type of adjacent buildings $\left(P_{4}\right)$, the possibility of vertical fire propagation $\left(P_{5}\right)$, the building conservation status $\left(P_{6}\right)$, the existence of fire breaks/buffer zones $\left(P_{7}\right)$ according to:

$$
G_{1}=B P=\left[\operatorname{Max}\left(P_{i, i=1: 7}\right)\right] \times 0.35
$$

For this simplified assessment, $P_{1}$ is defined using values from the literature for historical constructions as the sum of two components: the immovable load density $I_{q}$ and the movable contents $M_{q}$ related to the building use. Parameter $I_{q}$ is defined by the combustibility of constructive materials. Combustible materials are, for example, timber, textile, organic materials, plastics, resin steel or stucco. Based predominant combustib assessment (Table 1). Parameter $\boldsymbol{M}_{q}$ is determi (Table 2). These and other values can be found ir
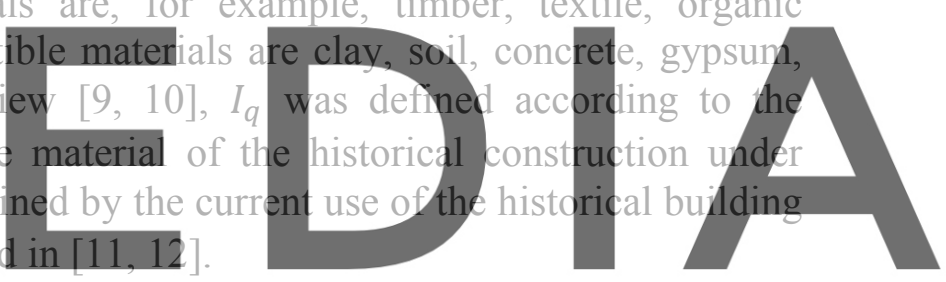

Table 1: Values proposed for parameter $I_{q}$

Register for free at https//www.scipedia.com to download the version without the watermark Type of structure (Finishes and Supports) Fire Load Structure made with a least $80 \%$ of combustible materials, e.g. floors $\quad 3,000 \mathrm{MJ} / \mathrm{m}^{2}$ and walls made of timber.

Structure made with a balance of non-combustible and combustible $\quad 1,100 \mathrm{MJ} / \mathrm{m}^{2}$ materials, e.g. masonry walls and timber floors or thatch roofs.

Structure made with at least $80 \%$ of non-combustible materials, e.g. $\quad 200 \mathrm{MJ} / \mathrm{m}^{2}$ masonry walls and reinforced concrete slabs.

Table 2: Values proposed for parameter $M_{q}$ according to the building activity.

\begin{tabular}{cccc}
\hline $\begin{array}{c}\text { Type of Use } \\
\text { (Current activity) }\end{array}$ & Fire Load & Type of Use (Current activity) & Fire Load \\
\hline Housing/Apartment/Dwelling & $780 \mathrm{MJ} / \mathrm{m}^{2}$ & Officine & $1,200 \mathrm{MJ} / \mathrm{m}^{2}$ \\
\hline Museum/Art galleries & $300 \mathrm{MJ} / \mathrm{m}^{2}$ & Hotels, Hospitals & $300 \mathrm{MJ} / \mathrm{m}^{2}$ \\
\hline Churches (furniture and candles) & $1,300 \mathrm{MJ} / \mathrm{m}^{2}$ & School & $300 \mathrm{MJ} / \mathrm{m}^{2}$ \\
\hline Libraries/Archives & $1,500 \mathrm{MJ} / \mathrm{m}^{2}$ & Shopping/Cinema/Theatre & $800 \mathrm{MJ} / \mathrm{m}^{2}$ \\
\hline
\end{tabular}

Parameter $P_{2}$ is defined based on $[13,14]$ to be consistent with the European Reaction to 
Fire classification system. The classification is based on the predominant material as for $I_{q}$, and its susceptibility to produce flashover according to seven types (A1, A2, B, C, D, E and F). From A1 to F, materials have a decreasing fire resistance, thus a higher likelihood of producing a flashover. Parameter $P_{3}$ is related to the possibility of containing the occurrence of a fire within certain areas of the building to avoid its propagation, and reflects the size of the largest fire compartment with combustible or flammable materials. The lack of containment elements between compartments (i.e. doors) can lead to an increase of the class of $P_{3}$ by one level. The classification of this parameter is based on criteria defined in [7, 14] but accounts for other features such as the existence of self-closing doors or windows. When there are self-closing systems, the classification of $P_{3}$ can be reduced by one level (e.g. if the compartmentalisation classification leads to Class B, it can be upgraded to Class A if there are self-closing systems).

Parameter $P_{4}$ reflects the influence of adjacent buildings according to their location and type of construction (i.e. in terms of combustible materials). Following the distance criteria in [15],

$P_{4}$ is classified as type IS if the construction under assessment has no surrounding constructions with exposed combustible materials closer than $12 \mathrm{~m}$, made of timber closer than $6 \mathrm{~m}$, or made of quasi-fire resistant materials closer than $3 \mathrm{~m}$. Otherwise, $P_{4}$ is classified as type IC. $P_{4}$ can also be classified as AC if there are adjacent constructions made mostly with combustible materials, as ACN if there are adjacent constructions made with a balance of combustible and non-combustible materials, or as AN if there are adjacent constructions made with noncombustible materials. When the adjacent constructions are abandoned, $P_{4}$ is increased by one level. The classification within the construction of vertical aligned open levels between opening height of the opening 0 analyse the relation betw
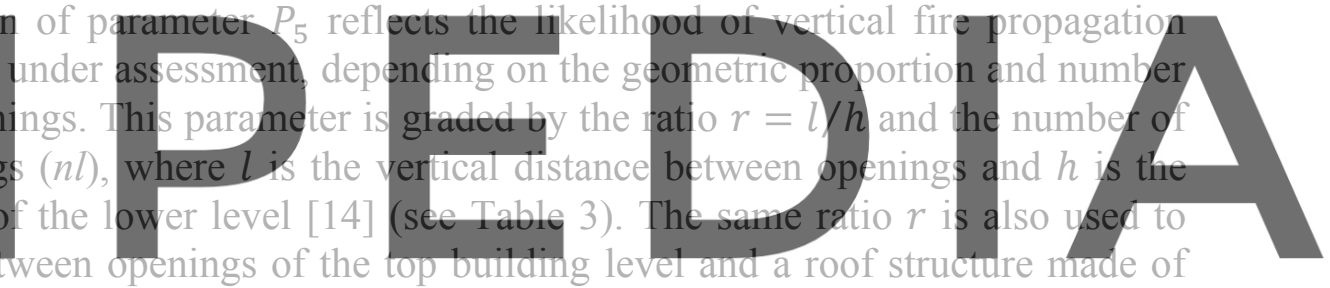
combustible material. The classification of $P_{5}$ can be reduced by one level if the construction

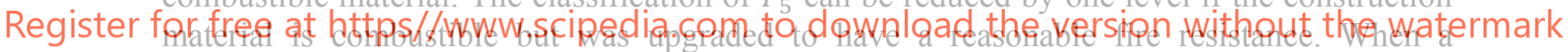

construction presents situations with both $r \geq 1$ and $r<1, P_{5}$ can be graded as $\mathrm{C}$, regardless of the number of levels. In case there are no aligned openings and roofs have non-combustibie materials (NAI), $P_{5}$ can be graded as A. Parameter $P_{6}$ reflects the level of conservation and cleanliness of the construction's combustible materials (e.g. timber, thatch, or bamboo). This parameter is graded according to five levels ranging from Very Good (when there are no combustible materials in the building), Good (when the combustible materials are in good condition and have a fire-resistance finishing), Medium (when the combustible materials appear to be in good condition, without any type of biological colonisation (i.e. termites) and high porosity), Bad (when the materials exhibit high porosity produced by biological colonisation), and Very Bad (when the combustible materials exhibit high concentration of humidity, biological colonisation, porosity, and vegetation growth; a common situation in abandoned historical constructions). Parameter $P_{7}$ reflects the horizontal propagation of fire from exterior combustible materials through the distance between the construction under assessment and the surrounding vegetation, rubbish or other elements that may exist. In the case of surrounding vegetation, based on criteria from the United States National Fire Protection Association and recommendations from the Portuguese Civil Protection, $P_{7}$ was graded from A to $\mathrm{E}$ when the 
referred distance is more than $50 \mathrm{~m}$, between $50 \mathrm{~m}$ and $30 \mathrm{~m}$, between $30 \mathrm{~m}$ and $5 \mathrm{~m}$, from $5 \mathrm{~m}$ to $1.5 \mathrm{~m}$, and less than $1.5 \mathrm{~m}$, respectively. $P_{7}$ is also associated with the criteria used by [15] which involve the critical distance between constructions made of combustible materials defined by types I to IV. In this case the critical distance is defined with respect to other possible exterior combustible materials, namely street furniture, rubbish or other elements that are not vegetation or other constructions. Types I to IV correspond to cases where the distance from ignition sources is more than $12 \mathrm{~m}$, between $12 \mathrm{~m}$ and $6 \mathrm{~m}$, between $6 \mathrm{~m}$ and $3 \mathrm{~m}$, and less than $3 \mathrm{~m}$, respectively. The classification of $P_{7}$ can be reduced by one level if the heritage asset near combustible or flammable materials is made of non-combustible materials. Table 3 summarizes the classifications of indicators $P_{1}$ to $P_{7}$.

Table 3: Classification of parameters $P_{1}$ to $P_{7}$.

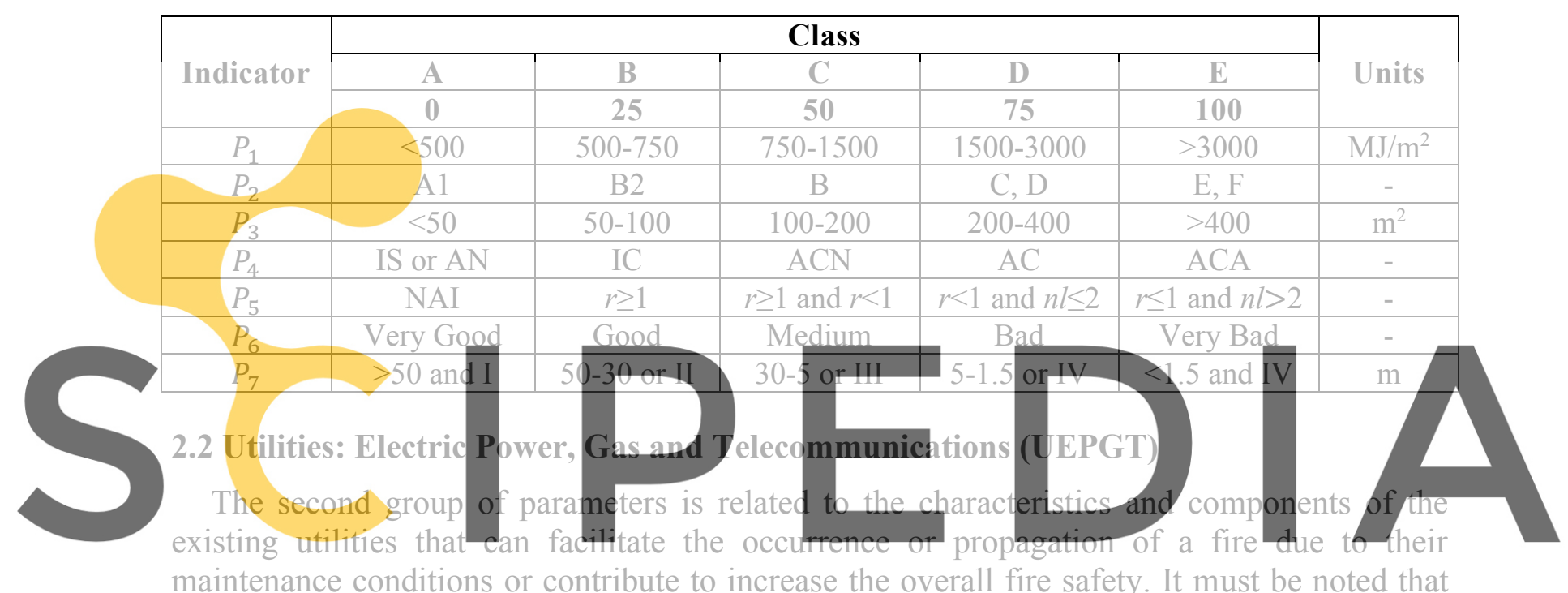

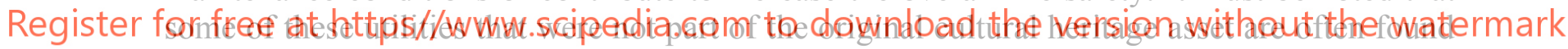

to be operating under inadequate safety conditions (e.g. unprotected electrical wiring or electrical overload of power outlets). These unsafe conditions increase the vulnerability of the construction and its contents. The UEPGT group considers five indicators related to the electrical $\left(P_{8}\right)$, gas $\left(P_{9}\right)$, HVAC $\left(P_{10}\right)$, telecommunications and CCTV systems $\left(P_{11}\right)$, to the technical control room $\left(P_{12}\right)$, and that are combined according to:

$$
G_{2}=U E G P T=\left[\operatorname{Max}\left(P_{i, i=8: 12}\right)\right] \times 0.20
$$

The classification of parameter $P_{8}$ follows principles referred in $[6,16]$ to reflect the level of maintenance of the electrical installation. The grading levels (see Table 5) are Excellent (EX) (adequate isolation of electrical components and combustible materials installed by professionals), Good (G) (refers to installations similar to EX but not installed by professionals), Medium (M) (installation with both new and older electrical components), Bad (BA) (installation with older electrical components only), and Very Bad (VB) (installation similar to BA but with known electrical deficiencies). The deficient implementation of a lightning rod in buildings with tall vertical elements (e.g. a bell tower, pinnacle or cupola) can also be considered to increase the classification of $P_{8}$ by one level [17]. Parameter $P_{9}$ is graded 
in Table 4 according to the type of gas supply [6], which can be piped (P), a gas reservoir (GR), an external gas container (EGC), an internal gas container in a well-ventilated area (IGCV), or an internal gas container in a poorly-ventilated area or close to heat sources (IGCNV). The absence of gas installations is graded as A. The classification of $P_{9}$ is increased one level when gas installations or gas heaters are near flammable materials. Parameter $P_{10}$ reflects the existence of an HVAC system, that could be decentralized (D) or centralized (C). If the system exhibits a good working condition $P_{10}$ is graded as B (see Table 4). If the system's level of maintenance is inadequate, the classification increases to $\mathrm{C}$ for a decentralized system (DCF) and to $\mathrm{D}$ for a centralized system (CCF). These classifications increase one more level if the system is near combustible or flammable materials (to LMD and LMC for decentralized and centralized systems, respectively). The absence of HVAC (NH) is equivalent to Class A. Parameter $P_{11}$ combines information related to the likely level of criminality of the area of the construction under assessment and to the availability of systems with automatic communication with the fire brigade services and video surveillance (CCTV). Parameter $P_{11}$ is thus graded in Table 4 as TT1 when there is a good CCTV system in low criminality zones, TT2 when there is a good CCTV system in moderate or high criminality zones, TT3 when there is an incomplete CCTV system (i.e. cameras are not available in all the relevant spaces), and TT4 when there is no adequate CCTV system. The TT1-TT4 grading assume there is an efficient communication system with the fire brigade. If this condition is not met, the classification can increase one level. A grading TT5 is assigned when there is no CCTV system and no automatic

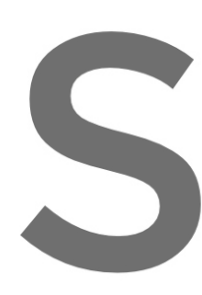
communication systen

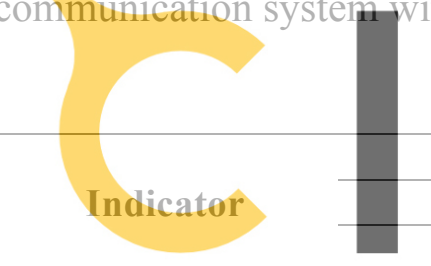

$P_{8}$
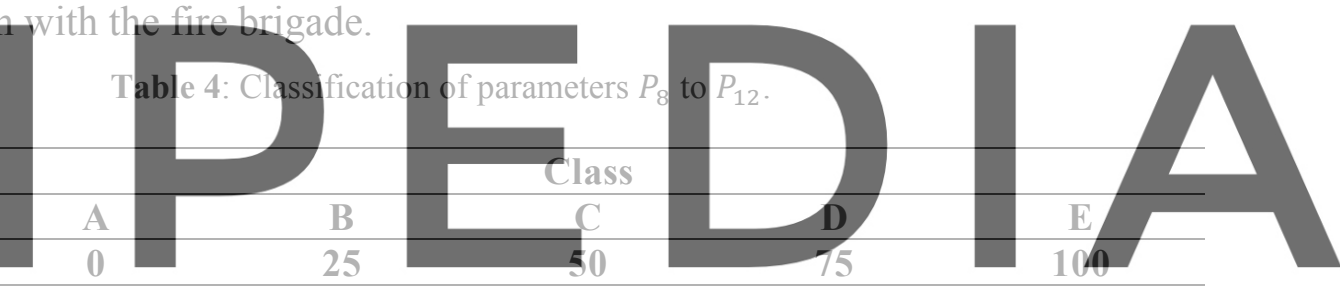

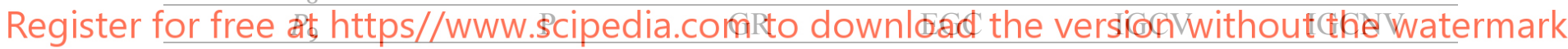

\begin{tabular}{cccccc}
\hline$P_{10}$ & NH & D or C & DCF & CCF or LMD & LMC \\
\hline$P_{11}$ & TT1 & TT2 & TT3 & TT4 & TT5 \\
\hline$P_{12}$ & Out $\geq 5$ & Out $<5$ & IMC & IMM & IB \\
\hline
\end{tabular}

Due to the flammability of components that a technical control room can have, its contribution to the impacts is assessed independently by $P_{12}$ (Table 5). This parameter reflects the location of the technical control room within the construction under assessment, its degree of maintenance, and the fire-resistant nature of the surface materials. When the technical control room is located outside the main construction at a distance more than $5 \mathrm{~m}, P_{12}$ is assigned to Class A, whereas when the engine room is located at a distance equal or less than $5 \mathrm{~m}, P_{12}$ is assigned to Class B. Assigning $P_{12}$ to Class C (IMC) corresponds to cases where the technical control room is inside the construction and exhibits a good condition and there are some fire control systems in place (e.g. an active suppression system, fire-retardant materials, or selfclosing doors). If the technical control room is located inside the construction and exhibits a good condition but there are no active or passive fire suppression systems, class D (IMM) is assigned. If the technical control room does not exhibit a good condition or there are no fire suppression systems, $P_{12}$ is assigned to class E (IB). If there are flammable elements or 
combustible materials that are not associated to the functionality of the technical control room (e.g. textiles, mattresses, candles, ropes), the classification of $P_{12}$ is increased one level.

\subsection{Firefighting measures (FM)}

The firefighting measures involves five parameters graded according to Table 5 and combined according to Equation 4. This group addresses the existence of alarm and detection systems $\left(P_{13}\right)$, of smoke control systems $\left(P_{14}\right)$, of active suppression systems $\left(P_{15}\right)$, of water supply systems $\left(P_{16}\right)$, and the site accessibility by and its proximity to fire rescue services $\left(P_{17}\right)$.

$$
G_{3}=F M=\left[\operatorname{Max}\left(P_{i, i=13: 17}\right)\right] \times 0.30
$$

Parameter $P_{13}$ reflects the existence of an efficient alarm and fire detection system where all components are in good working condition (except when stated otherwise). The grading of $P_{13}$ involves the classes AM (when there is a dual (automatic and manual) system in all the relevant zones of the construction under assessment), AA (when there is only an automatic system), AMN (when there is a dual system with deficiencies in the automatic component), AAN (when there is only an automatic system that is not in perfect working conditions), and NAD (when there is no system). The classification of $P_{13}$ can increase one level when there is a significant likelihood of false alarms. Parameter $P_{14}$ refers to the existence of smoke control systems in enclosed compartments (automatic systems, through the spatial arrangement of the building, or both) that can facilitate evacuation or firefighting actions [18]. Parameter $P_{14}$ is graded as SC1

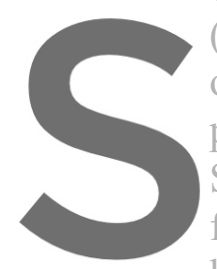
(when there are active and pasive systems in good working
only a functional active systen in corridors, stairs, or elevator
passive extraction systems), SC4 (when there is a partially w
SC5 (when there are no smoke control systems). Parameter $P_{1}$
fire suppression systems that have any of the following compd hydrants, hose reels, wet and dry rising mains, drencher systems, sparge pipes, water curtains, conditions), SC2 (when there is
shafts), SC3 (when there is oply
orking SC2 or SC3 system) and
i5 reflects the existence of active
anents. sprinklers, extinguishers,
ms, sparge pipes, water curtains,

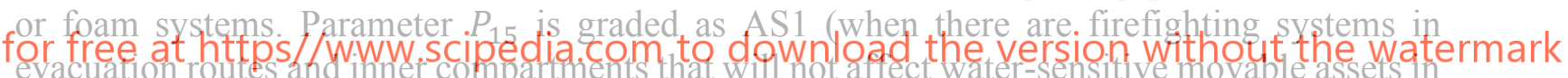
case of a fire), AS2 (when there are firefighting systems in locations that can affect watersensitive movable assets in case of a fire), AS3 (when there are only fire suppression systems in evacuation zones), AS4 (when there are suppression systems that have faulty or unreliable components), and AS5 (when there is no firefighting equipment). Parameter P16 addresses the water supply systems that include interior (e.g. water tanks) or exterior (e.g. fire hydrants, rivers, lakes, etc.) water sources. Parameter $P_{16}$ is graded as W1 (when the supply can be either from the interior of the construction under assessment with a well-designed water tank or from its exterior by a source with enough capacity (e.g. at least $20 \mathrm{~m}^{3}$ ), that is closer than $20 \mathrm{~m}$ ), W2 (when the source is from an interior water tank with insufficient capacity, and exterior sources closer than $10 \mathrm{~m}$ ), W3 (when all the possible exterior sources are between $10 \mathrm{~m}$ and $20 \mathrm{~m}$ away), W4 (the possible exterior sources are between 20 and 40m away), and W5 (when there are no interior or exterior sources for water supply). In situations where the exterior water sources are fundamental, and if their availability is known to be scarce or water is frozen in specific times of the year, the classification of $P_{16}$ should increase by two levels. When the fire suppression system presents an adequate automatic non-water-based alternative, $P_{16}$ can be graded as W1. With respect to parameter $P_{17}$, the selected criteria are based on the firefighters' expected time 
of arrival at the construction under assessment, partially based on [19] (see Table 5). When $P_{17}$ is graded as $\mathrm{E}$ or $\mathrm{D}$, this construction can be reduced two levels if $P_{13}, P_{15}$ or $P_{16}$ are of Class A (e.g. if the construction has AM, AS1, W1 and $P_{17}>40$ min, $P_{17}$ should be graded as C). The grade of $P_{17}$ increases one level if the access to the façade of the construction has physical obstacles, if there is a unique or no road access, or if the access routes have heavy traffic.

Table 5: Classification of parameters $P_{13}$ to $P_{17}$.

\begin{tabular}{|c|c|c|c|c|c|c|}
\hline \multirow{3}{*}{ Indicator } & \multicolumn{5}{|c|}{ Class } & \multirow{3}{*}{ Unit } \\
\hline & $\mathbf{A}$ & B & $\mathbf{C}$ & D & $\mathbf{E}$ & \\
\hline & 0 & 25 & 50 & 75 & 100 & \\
\hline$P_{13}$ & $\mathrm{AM}$ & AA & AMN & AAN & NAD & - \\
\hline$P_{14}$ & SC1 & $\mathrm{SC} 2$ & SC3 & $\mathrm{SC} 4$ & SC5 & - \\
\hline$P_{15}$ & AS1 & AS2 & AS3 & AS4 & AS5 & - \\
\hline$P_{16}$ & W1 & W2 & W3 & W4 & W5 & - \\
\hline$P_{17}$ & $0-10$ & $10-20$ & $20-30$ & $30-40$ & $>40$ & $\min$ \\
\hline
\end{tabular}

\subsection{Emergency Preparedness Planning (EPP)}

This group reflects the availability of measures and strategies implemented to safeguard the construction and its movable assets given their cultural value. This group integrates parameters addressing the existence of emergency planning $\left(P_{18}\right)$, the elevation of relevant compartments of the construction $(P$

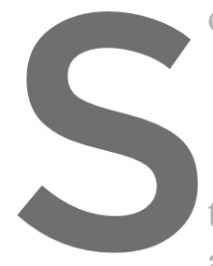
Parameter $P_{18}$ is graded as EA when the staff assets in case of fire and specific procedures for the fire brigade
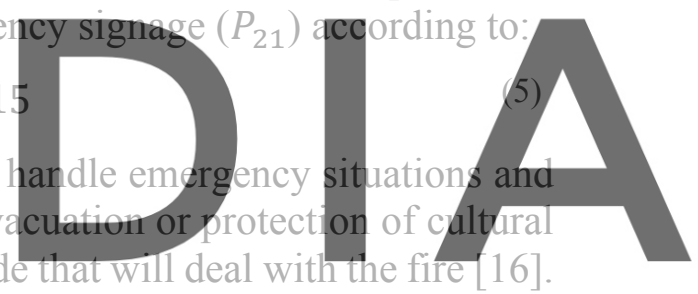

This emergency response plan needs to involve guidance for staff training, information about

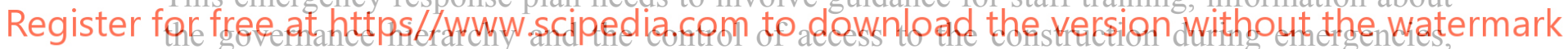

guidance for the evacuation of cultural assets during emergencies and procedures to carry out reguiar safety/risk assessments. The emergency pian, must have strategies to address scenarios where rehabilitation or refurbishment operations that may involve hot works are carried out [18]. If the emergency plan only accounts for the safety of people, $P_{18}$ is graded as EB. $P_{18}$ is graded as EC when the staff is trained to handle emergency situations, but there is no emergency plan. P18 is graded as ED when there is an emergency plan but the staff is not trained to handle emergencies. If there is no emergency plan and if the staff is not trained to handle emergencies, $P_{18}$ is graded as EE. Parameter $P_{19}$ is linked to the elevation of the habitable space (HS) or the compartments with cultural assets (CUA), considering the most disadvantageous case (see Table 6). The classification of $P_{19}$ can be modified when CUA and/or HS are located at a height over $9 \mathrm{~m}$ but there are active suppression systems (AS1 or AS2) and when efficient strategies of staff training are also integrated into the emergency plan (EA) [18]. Parameter $P_{20}$ combines multiple factors (i.e. travel distance to safety points $(d)$, the width of corridors $(w)$ and their slope $(s)$ ) based on $[5,16]$, to reflect the characteristics of the evacuation routes relevant for the evacuation of movable assets (from A to E), or for people (from B to E). If $P_{20}$ is classified as $\mathrm{D}$ or $\mathrm{E}$, it can be improved by one level (up to three) each time one of the following are 
available: efficient active suppression systems (AS3), smoke control systems (SC1, SC2 or $\mathrm{SC} 3$ ) and EA in evacuation zones or specific meeting points. On the other hand, $P_{20}$ is increased by one level when there are combustible materials obstructing evacuation routes. Parameter $P_{21}$ considers the contribution of signage and emergency lights to the safeguard of movable assets and the safety of people during an emergency. When there are emergency lights in the evacuation routes (EL) and the signage provides instructions for both the safeguard of movable assets (SM) and the safety of people (SP) during an emergency, $P_{21}$ is of class A. When there are emergency lights in the evacuation routes (EL) but the signage only addresses the safety of people (SP), $P_{21}$ is of class B. When there is only one element (i.e. emergency lights or signage) that provides the safeguard of movable assets (SM) and the safety of people (SP) during an emergency, $P_{21}$ is of class C. When there is only signage addressing the safety of people (SP), $P_{21}$ is of class D. When there are no emergency lights and signage $(\mathrm{N}), P_{21}$ is of class E. If the emergency lights are not in good working condition, $P_{21}$ increases by one level.

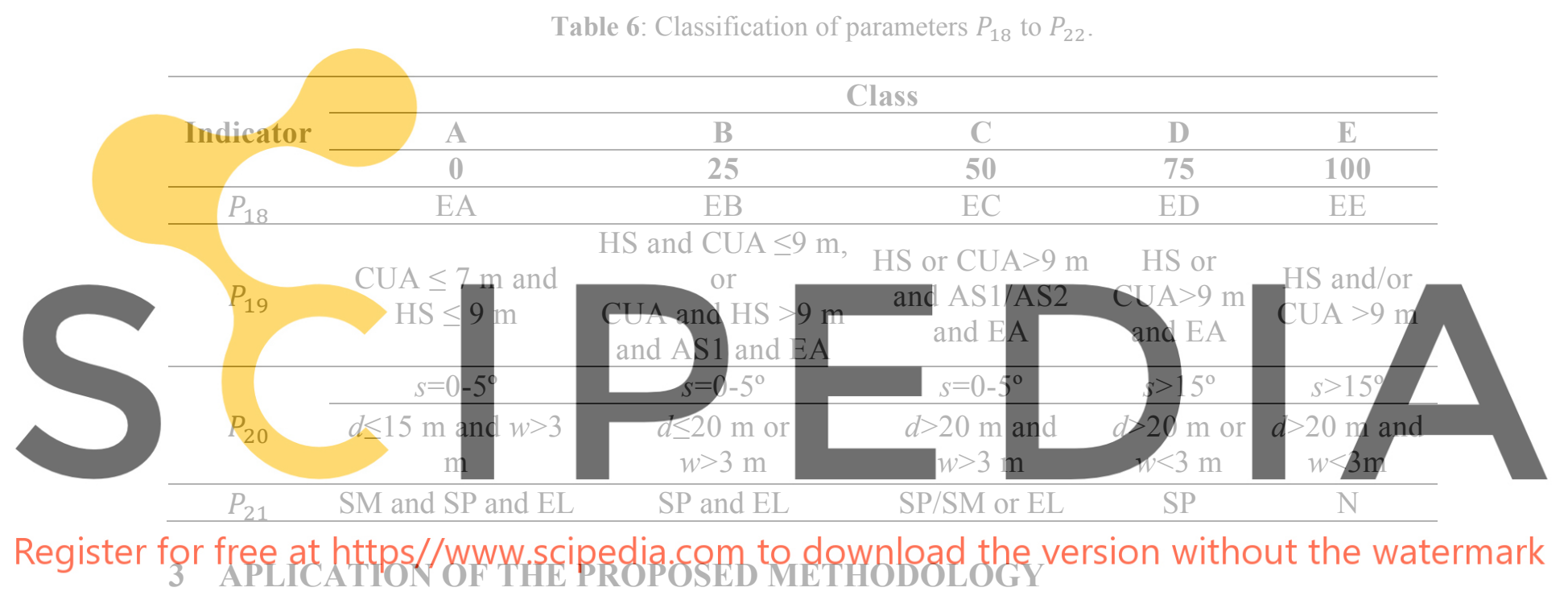

\subsection{Description of the case studies}

The first case study is the $16^{\text {th }}$ century Church of Misericordia in Esposende, Portugal, $\left(41^{\circ} 31^{\prime} 51.85^{\prime \prime} \mathrm{N}, 8^{\circ} 46^{\prime} 50.18^{\prime \prime} \mathrm{W}\right)$. Based on data available from an in-situ survey, the BP group was classified as D. The church exhibits a balance between combustible (i.e. main and secondary altars, roof, mezzanine and stairs made of timber (see Figure 1a)) and noncombustible materials (i.e. stone walls), and its current use is religious with an archive, thus leading to an estimated fire load for $P_{1}$ of $2600 \mathrm{MJ} / \mathrm{m}^{2}$ (Class D). $P_{2}$ was graded as $\mathrm{C}$, considering since the unprotected timber, namely, in the altars and the furniture, is a fire susceptible material. $P_{3}$ is graded as $\mathrm{C}$ since the largest compartment size is approximately 103 $\mathrm{m}^{2}$, whereas $P_{4}$ is equal to A since the church is an isolated construction. The ratios $r$ that were evaluated lead to a $P_{5}$ of Class C. Due to the level of degradation exhibited by some combustible materials and the lack of intumescent materials, $P_{6}$ is graded as C. For $P_{7}$, E was assigned due to a tree located approximately $4.48 \mathrm{~m}$ from one of the entrances (see Figure $1 \mathrm{~b}$ ). UEGPT was assigned to Class $\mathrm{E}$ due to $P_{8}$, and $P_{12}$. The former is graded as $\mathrm{E}$ because there are extension cords close to the combustible materials behind the main altar (see Figure 1c), and there are 
frequent power surges when many electric components are turned on. The existence of gas containers stored in a poorly-ventilated area inside the building (see Figure 1d) and the use of gas heaters during ceremonies leads to a class D for $P_{9}$. The building does not have a HVAC system, leading to $P_{10}$ of Class A. Since the building has a few cameras in the interior, $P_{11}$, is graded as C. $P_{12}$ was graded as $\mathrm{E}$ due to a lack of fire suppression systems and the fact there is a mattress located in the compartment of the electrical control panel. FM was assessed as $\mathrm{E}$ due to a lack of fire detection $\left(P_{13}\right)$, smoke control $\left(P_{14}\right)$ and fire suppression systems $\left(P_{15}\right)$. Still, $P_{16}$ is graded as $\mathrm{C}$ (there is an exterior fountain $10 \mathrm{~m}$ away and a public hydrant at approximately $20 \mathrm{~m}$ ), and $P_{17}$ is graded as B (the firefighter services are nearby). Even though the height of the upper level is lower than $7 \mathrm{~m}\left(P_{19}\right.$ is $\left.\mathrm{A}\right)$, EPP was graded as E due to a lack of emergency planning (Class E), the travel distance being more than 20m (Class E), and the door widths being lower than $3 \mathrm{~m}$ (Class $\mathrm{E}$ ). The absence of signage and the presence of emergency lights that are not in good working conditions lead to $P_{21}$ of Class D. Table 7 and Figure 3 a summarize the assessed parameters and present a final damage level $D_{V}$ of 91.25 (heavy damage).

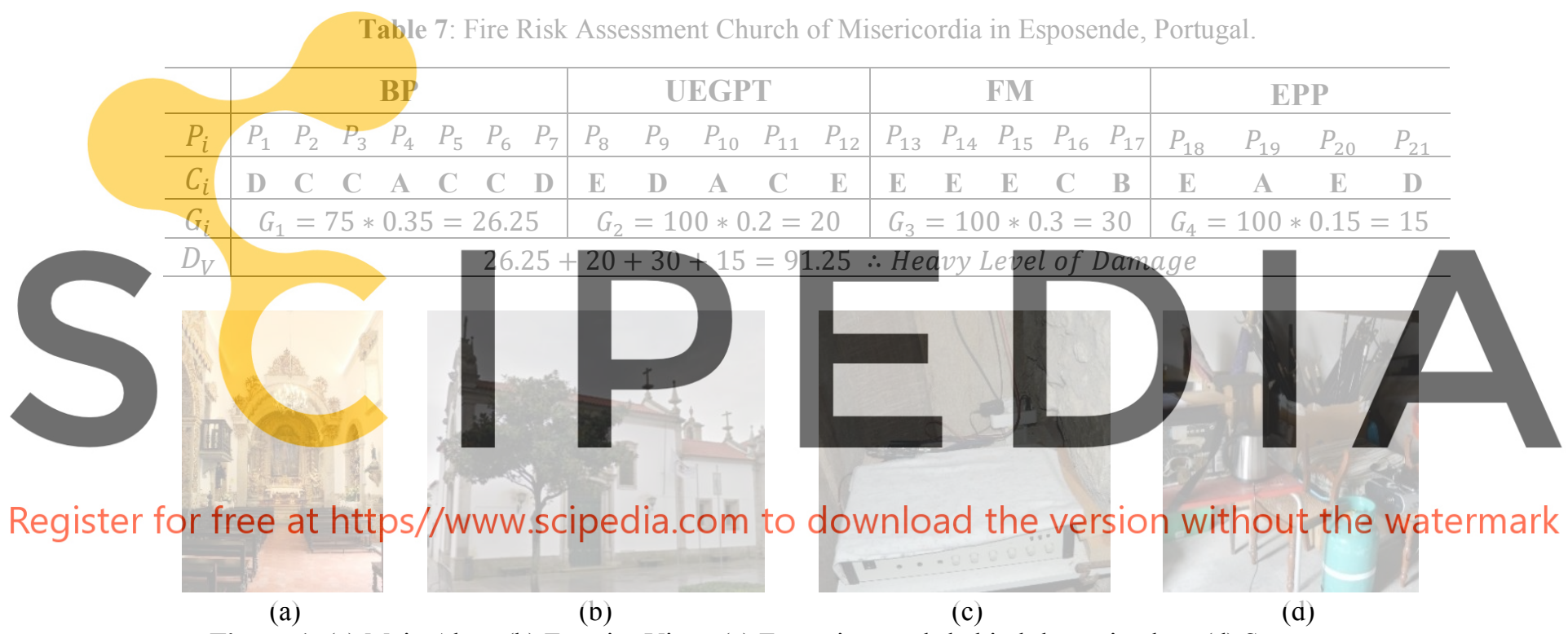

Figure 1: (a) Main Altar; (b) Exterior View; (c) Extension cords behind the main altar; (d) Storage room.

The second case is the $14^{\text {th }}$ century Museum of Carmo in Lisbon, Portugal $\left(38^{\circ} 42^{\prime} 43.5^{\prime \prime} \mathrm{N}\right.$ $\left.9^{\circ} 08^{\prime} 25.4^{\prime \prime W}\right)$. The BP group was ranked as D, given that, in $P_{5}, r<1$ and $n l \leq 2$. $P_{3}$ was graded as $\mathrm{C}$ since the largest compartment has approximately $190 \mathrm{~m}^{2} . P_{1}$ and $P_{7}$ were graded as $\mathrm{B}$ due to the current use of the building (being a museum $80 \%$ with non-combustible material leads to a fire load of $500 \mathrm{MJ} / \mathrm{m}^{2}$ ) and the existence of vegetation 5-30m away from non-combustible materials, respectively. The other parameters of BP were graded as $\mathrm{A}$, due to the noncombustible materials of the walls and the type of adjacent buildings $\left(P_{2}\right.$ and $\left.P_{4}\right)$, and the good level of conservation of the few combustible materials in the structure $\left(P_{6}\right)$. In terms of UEGPT, the building was graded as B, given that utilities are in good condition, the technical room is in the exterior but near the administrative zones, there is a CCTV system (Figure 2b) and the building is in a moderate criminal area. FM is graded as $\mathrm{C}$ since there is a fire suppression system (Figure 2a), an alarm and detection system (Figure 2b), a passive smoke control system (atrium), water supply and the firefighters are less than 10min away. Even though $P_{20}$ leads to 
a Class D (travel $d<20 \mathrm{~m}$ and $w<3$ ), it is upgraded to B due to the integration of AS1 and SC3. EPP is graded as $\mathrm{C}$ since there are some measures of emergency preparedness but no emergency plan, given that the elevation is lower than $7 \mathrm{~m}$ and that there are signage and emergency lights, (see Figures 2c and 2d). The summary of the parameters is shown in Table 8 and Figure $3 b$, leading to a final damage level of 53.75 (medium damage).

Table 8: Fire Risk Assessment of Museum of Carmo Convent in Lisbon, Portugal.

\begin{tabular}{|c|c|c|c|c|}
\hline & BP & UEGPT & FM & EPP \\
\hline$P_{i}$ & $\begin{array}{lllllll}P_{1} & P_{2} & P_{3} & P_{4} & P_{5} & P_{6} & P_{7} \\
\end{array}$ & $\begin{array}{lllll}P_{8} & P_{9} & P_{10} & P_{11} & P_{12} \\
\end{array}$ & $\begin{array}{lllll}P_{13} & P_{14} & P_{15} & P_{16} & P_{17} \\
\end{array}$ & $\begin{array}{llll}P_{18} & P_{19} & P_{20} & P_{21}\end{array}$ \\
\hline$C_{i}$ & $\begin{array}{lllllll}\mathbf{B} & \mathbf{A} & \mathbf{C} & \mathbf{A} & \mathbf{D} & \mathbf{A} & \mathbf{B}\end{array}$ & $\begin{array}{lllll}\mathbf{A} & \mathbf{A} & \mathbf{A} & \mathbf{B} & \mathbf{B} \\
\end{array}$ & $\begin{array}{lllll}\mathbf{A} & \mathbf{A} & \mathbf{C} & \mathbf{A} & \mathbf{A} \\
\end{array}$ & $\mathrm{C}$ \\
\hline$G_{i}$ & $G_{1}=75 * 0.35=26.25$ & $G_{2}=25 * 0.2=5$ & $G_{3}=50 * 0.3=15$ & $G_{4}=50 * 0.15=7.5$ \\
\hline$D_{V}$ & 26.25 & $+15+7.5=53.75$ & Medium Level of $\mathrm{Da}$ & \\
\hline
\end{tabular}

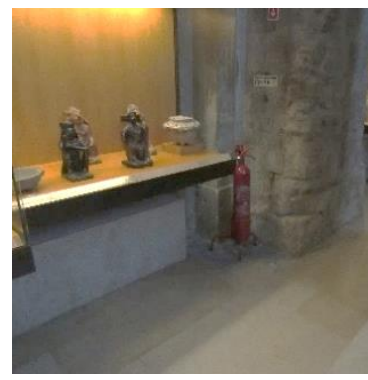

(a)

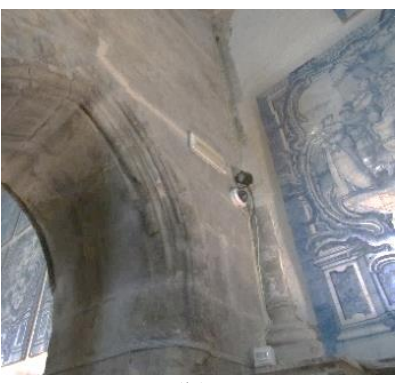

(b)

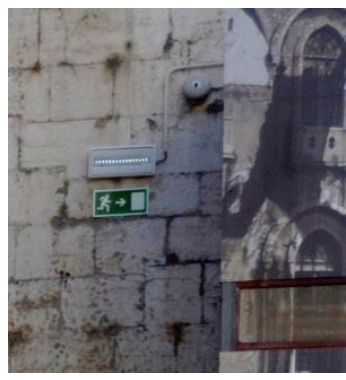

(c)

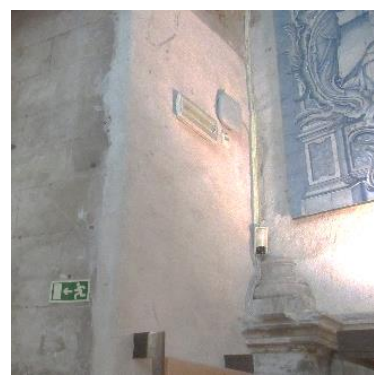

(d)

Figure 2: (a) Suppression System; (b) CCTV; (c) Alarms system; (d) Signage.

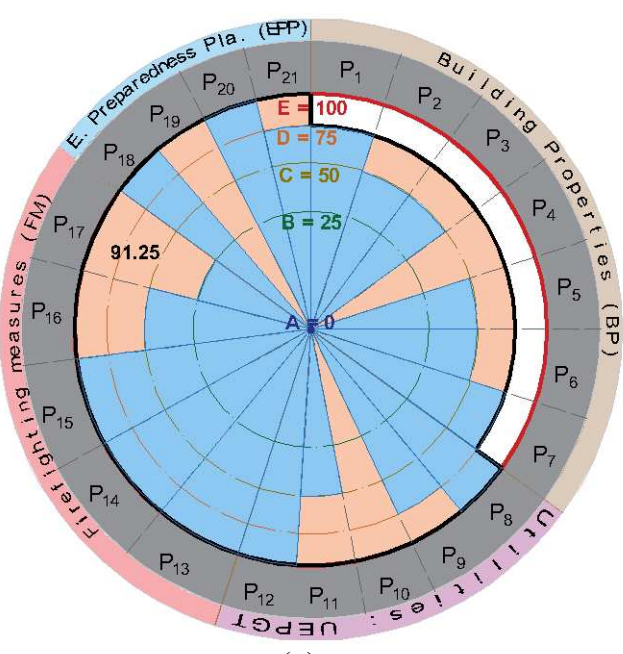

(a)

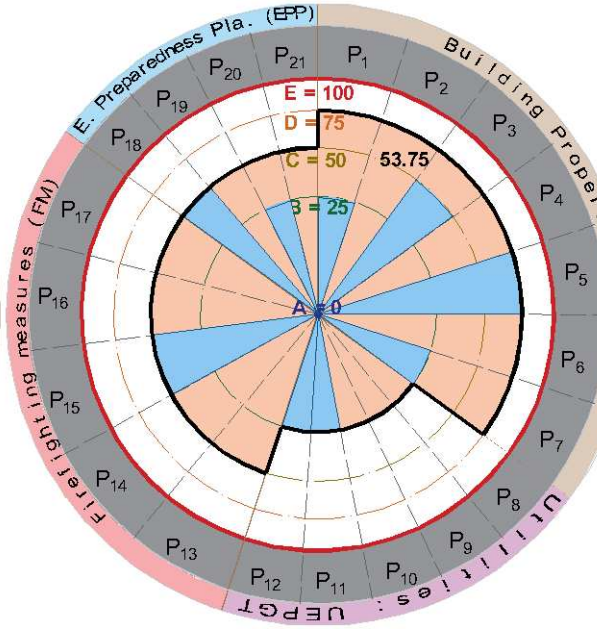

(b)

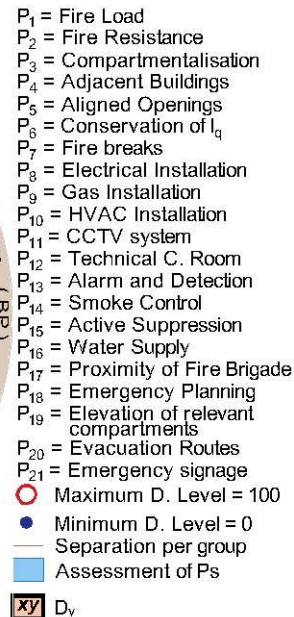

Xy $D_{r}$

Figure 3: Fire index assessment (a) $16^{\text {th }}$ Church of Misericordia; (b) $14^{\text {th }}$ century Museum of Carmo.

\section{CONCLUSIONS}

Given the diversity and specificity of cultural heritage assets, simple approaches are required to analyse their fire safety and establish their risk mitigation needs. In this context, a simplified fire damage index dedicated to assessing the expected damage impact due to fires in immovable cultural heritage assets is proposed. The proposed index involves a weighted multi-parameter 
evaluation that can be correlated with the level of damage that the cultural heritage asset (including its contents) is expected to suffer under a fire. The fire index was applied to two case studies in Portugal to illustrate the flexibility of its applicability.

Acknowledgements. The authors acknowledge the support of the BD scholarship funded by FCT (Fundação para a Ciência e a Tecnologia) with reference PD/BD/150405/2019, the project POCI-01-0145-FEDER-030469 - RIACT - Risk Indicators for the Analysis of Cultural heritage under Threat, funded by FEDER funds through COMPETE2020 - POCI and by national funds through FCT, and the Base Funding UIDB/04708/2020 of CONSTRUCT - Instituto de I\&D em Estruturas e Construções, funded by national funds through FCT/MCTES (PIDDAC).

\section{REFERENCES}

[1] Wen, I., Lee M. and Wu, S. Factors Affecting Fire Risk Assessment of Historic Buildings in Taiwan, J. Applied Fire Science, 4 (2013) 22:447-460

[2] NFPA, Code for the Protection of Cultural Resource Properties - Museums, Libraries, and Places of Worship (2013).

[3] Watts, J. and Kaplan, M. Fire Risk Index for Historic Build., F. Tech. (2001)37:167-180.

[4] Chichorro, M., Ferreira, R. and Correia, A. Risk Assessment of Urban Fire - Proposal of a Model for Analysis and Management of Existing Building. (2016).

[5] Lopes, J. P., Coelho, A. L. and Rodrigues J. P. C. Urban Fire Risk Evaluation and the Municipal Emergency Plans. (2012).

[6] Granda, S. and Ferreira, T. M. Assessing Vulnerability and Fire Risk in Old Urban Areas: Application to the Historical Centre of Guimarães. Fire Technology (2019) 55:105-127.

[7] Arborea, A., Mossa, G. and Cucurachi. Preventive fire risk assessment of Italian architectural heritage: An index-based approach. Key Eng. Mat. (2015) 628:27-33.

[8] Romão, X., Paupério, E. and Pereira, N. A framework for the simplified risk analysis of cultural heritage assets. Journal of Cultural Heritage. (2016) 20:696-708.

[9] Claret, M. and Andrade, A. Fire Load Survey of Historic Buildings: A Case Study. Journal of Fire Protection Engineering. (2007) 17:103-112.

[10] Li, J., Li, H., Zhou, B., Wang, X. and Zhang, H. Investigation and Statistical Analysis of Fire Loads of 83 Historic Buildings in Beijing. Int. J. of Architectural Her. (2018) 1-12.

[11] Coelho, A.L. Incêndios em edificios, Orion (2010).

[12] EN1991-1-2. Eurocode 1: Actions on structures - Part 1-2: General actions - Actions on structures exposed to fire. (2002).

[13] Hakkarainen,H and Y. Hayashi, Comparison of Japanese and European Fire Classification Systems for Surface Linings Fire Sci. \& Technology , 1 (2001) 21:19-42.

[14] Karlsson, B. Fire Risk Index Method - Multistorey Apart. Build. FRIM-MAB, V.1.2, (2000).

[15] Matsumiya and Oikawa. Quantitative Analysis of Fire Spreading Potential for Surrounding Areas of Cultural Properties in Kyoto City. J. of Asian A. and B. Eng. (2013) 12:269-276.

[16] CFPA-E, Managing fire safety in historical buildings, (2013).

[17] Rager, G. La conservation des objets mobiliers dans les églises La conservation des objets mobiliers dans les églises, Optimisa, (2004).

[18] Kidd, S. Fire Safety Management in Traditional Buildings. Technical applications and management solutions, Technical Conservation Group Historic Scotland, (2010).

[19] NFPA, Code for Fire Protection of Historic Structures, USA: NFPA Association, (2015). 